\title{
Incidence and risk factors for Dengue virus (DENV) infection in the first 2 years of life in a Brazilian prospective birth cohort
}

\author{
P. M. S. CASTANHA ${ }^{1,2} *$ U. R. MONTARROYOS ${ }^{2}$, S. M. M. SILVEIRA ${ }^{3}$, \\ G. D. M. AlBUQUERQUE ${ }^{1}$, M. J. G. MELLO ${ }^{3}$, K. G. S. LOPES ${ }^{1}$, \\ M. T. CORDEIRO ${ }^{1}$, E. T. A. MARQUES JR ${ }^{1,4,5}$, C. M. T. MARTELLI ${ }^{1}$ AND \\ C. BRAGA ${ }^{1,3}$ \\ ${ }^{1}$ Aggeu Magalhães Research Center, Oswaldo Cruz Foundation, Recife, Brazil \\ ${ }^{2}$ Biological Science Institute, Faculty of Medical Science, University of Pernambuco, Recife, Brazil \\ ${ }^{3}$ Instituto de Medicina Integral Prof. Fernando Figueira, Recife, Brazil \\ ${ }^{4}$ Center for Vaccine Research, University of Pittsburgh, Pennsylvania, USA \\ ${ }^{5}$ Department of Infectious Disease and Microbiology, University of Pittsburgh, Pennsylvania, USA
}

Received 21 February 2017; Final revision 1 August 2017; Accepted 29 August 2017; first published online 18 September 2017

\section{SUMMARY}

This study assessed the incidence and risk factors for dengue virus (DENV) infection among children in a prospective birth cohort conducted in the city of Recife, a hyperendemic dengue area in Northeast Brazil. Healthy pregnant women $(n=415)$ residing in Recife who agreed to have their children followed were enrolled. Children were followed during their first 24 months of age (May/2011-June/2014), before the 2015 Zika virus outbreak. DENV infection was detected by reverse-transcriptase polymerase chain reaction and/or serology (anti-DENV IgM/IgG).

The incidence rates per 1000 person-years (py) and its association with risk factors by age bands $(0-12,>12-30$ months) were estimated through Poisson regression models. Forty-nine dengue infections were detected; none progressed to severe forms. The incidence rates were 107.6/1000py (95\% CI 76.8-150.6) and 93.3/1000py $(95 \%$ CI 56.1-154.4) in the first and second years of age, respectively. Male children (risk ratios $(\mathrm{RR})=2 \cdot 33$; 95\% CI 1.09-4.98) and those born to DENV-naïve mothers $(\mathrm{RR}=2 \cdot 42 ; 95 \%$ CI $1 \cdot 01-5 \cdot 80)$ were at greater risk of infection in the first year of age. In the second year, children born to Caucasian/Asian descent skin colour mothers had a threefold higher risk of infection ( $R R=3 \cdot 34 ; 95 \% \mathrm{CI}: 1 \cdot 08-10 \cdot 33$ ). These data show the high exposure of children to DENV infection in our setting and highlight the role of biological factors in this population's susceptibility to infection.

Key words: Children, cohort, dengue virus, incidence.

\section{INTRODUCTION}

Dengue virus (DENV) infection is caused by one of the four distinct dengue serotypes (DENV-1-4) and

\footnotetext{
* Author for correspondence: P. M. S. Castanha, Aggeu Magalhães Research Center, Oswaldo Cruz Foundation, Av. Prof. Moraes Rego, s/n, Campus da UFPE, Cidade Universitária, Recife, Pernambuco, Brazil.

(Email: castanha.priscila@gmail.com)
}

transmitted by mosquitoes of the genus Aedes, usually Aedes aegypti [1,2]. Currently, more than half of the world's population is at risk of infection, and approximately 390 million dengue cases are estimated to occur annually throughout tropical and subtropical countries [3]. In Brazil, dengue incidence has dramatically increased in the last decade, and the country accounts for over $70 \%$ of the annually reported cases in the Americas [4, 5]. 
In Asian countries, severe dengue is one of the leading causes of hospitalization and death among infants and children [6-8]. Conversely, severe cases are more frequent among adults in Brazil [5]. These differences in the epidemiological pattern of dengue infections between regions highlight the diversity of risk factors involved in DENV transmission and morbidity.

Several risk factors in adult and paediatric populations, including individual (age, race, and low socioeconomic status) [9-12], household (intermittent water supply and unscreened houses) [12], environmental (inadequate garbage disposal) [9] and biological (immune status and genetic background) characteristics $[13,14]$, have been identified as predictors of dengue infection. In infants, epidemiological studies have provided evidence for a protective role of maternally transferred dengue antibodies against symptomatic DENV infection in the early months of life [15].

Prospective birth cohort studies provide a valuable tool for determining the incidence of DENV infection, risk factors for disease severity and decay of maternally transferred dengue antibodies in endemic settings $[16,17]$. These data are useful to estimate the force of infection of DENV transmission, to better understand the immunopathogenesis of severe dengue during infancy, and to establish an appropriate age for dengue vaccine schedules $[16,17]$.

In Brazil, despite the high transmission of the different DENV serotypes, dengue incidence and the risk factors associated with infection at early ages remains unknown. Therefore, we conducted a prospective birth cohort study in a large urban centre and hyperendemic dengue area in Northeast Brazil [18] to investigate the incidence of dengue infection and the kinetics of maternally transferred dengue antibodies among children in the first 2 years of life. We have previously described the high seroprevalence $(\sim 90 \%)$ of DENV-specific antibodies among pregnant women enrolled at baseline [19], the efficient placental transfer of dengue antibodies to neonates and the kinetics of decay of these antibodies in this cohort [20]. Here, we report the DENV incidence and associated risk factors for infection in this paediatric cohort.

\section{METHODS}

The cohort enrolment was performed in the maternity ward of the Instituto de Medicina Integral Professor Fernando Figueira (IMIP), a large publicly funded teaching hospital in the city of Recife (population: 1.7 million habitants), capital of Pernambuco state, between 2010 and 2012. Currently, the four DENV serotypes co-circulate in the city. DENV-1 was the first introduced serotype (1986), which was followed by DENV-2, DENV-3 and DENV-4 in 1995, 2002 and 2010, respectively [21]. Clinical and populationbased studies have confirmed the high endemicity of dengue in this setting [12, 21, 22]. During follow-up of the children (May 2011 and June 2014), all four DENV serotypes were detected in the city, and there was a predominance of DENV-1 between 2010 and 2011 and DENV-4 between 2012 and 2014 (Health Department of Recife, unpublished data).

The details of the study methodology were previously described [18]. Briefly, healthy pregnant women were enrolled at the time of admission for delivery. Low-risk pregnant women of any age residing in Recife and who agreed to have their child followed were invited to participate. Socio-demographic (maternal age, self-reported skin colour, schooling, and income) and prenatal information (report of dengue fever and infectious events during pregnancy) were obtained through a standardized questionnaire at enrolment. Maternal and umbilical cord blood samples were collected before and immediately after birth, respectively.

A total of 415 mothers (aged 13-43 years) and their newborns were enrolled at baseline [19]. The paediatric cohort was followed during the first 2 years of life. Children were randomly allocated into two different clinical visit schedules: Group 1 - months 2, 6, 10 and 18 and Group 2 - months 4, 8, 12 and 24. This follow-up strategy was adopted to minimize loss to follow-up due to repeated venous punctures. The strategy allowed for the collection of clinical data and blood samples every 2 months in the first year and at two different points (months 18 and 24) in the second year [18]. Losses to follow-up in the cohort were attributable to refusal in participate of the study and by difficulties in tracing cohort participants due to incorrect and/or change of residence address.

During the follow-up visits, children were examined by paediatricians who recorded clinical data with an emphasis on the history of acute febrile episodes. To track acute dengue infections, the parents were also instructed to contact the research team and bring their children for clinical examination if they presented with any febrile illness. Venous blood samples $(2 \mathrm{ml})$ were collected at each clinical visit and during any febrile acute episode to perform DENV serological and virological tests. The study was approved by the Ethics Research committees of CPqAM/ 
Fiocruz (59/10; CAAE-0061·0·095·000-10) and IMIP (n. 274426 December 2010).

\section{Laboratory procedures}

All serum samples from mothers and children were tested for DENV-specific immunoglobulin M (IgM) and $\mathrm{G}$ (IgG). Dengue indirect $\operatorname{IgG}$ (PanBio, Brisbane, Australia) and capture IgM (PanBio and Focus Diagnostics, Cypress, CA, USA) enzyme-linked immunosorbent assays (ELISA) were performed according to the manufacturer's instructions. Serum samples from children with acute febrile illness and/or clinical signs suggestive of dengue infection were also screened for dengue infection by reverse-transcriptase polymerase chain reaction (RT-PCR) according to a standardized protocol described elsewhere [19, 21]. Dengue serotype-specific neutralizing antibodies (DENV1-4) were assessed in sera from all mothers and DENV seropositive children by plaque reduction neutralization test (PRNT). PRNT was conducted in Vero cells, following a protocol described in details elsewhere $[20,22,23]$. The cut-off value for PRNT positivity was defined as $50 \%$ reduction $\left(\mathrm{PRNT}_{50}\right)$ in plaque counts at the lowest serum dilution used (1:20).

\section{Clinical and laboratory definitions}

DENV infection was confirmed as follows: (a) positive DENV RT-PCR result in the acute-phase sera from febrile children or (b) positive DENV-specific IgM result in any sample collected during follow-up, followed by DENV-specific IgG seroconversion. DENV-specific IgG seroconversion was defined according to the following criteria: (i) DENV-specific IgG positive at birth, followed by IgG negative result at any time point during follow-up and IgG seroconversion afterwards; (ii) DENV-specific IgG negative at birth, followed by IgG seroconversion.

DENV infection was classified as symptomatic when a child presented laboratorial evidence of dengue (IgM or RT-PCR) along with the report of current or previous acute febrile illness between birth and any follow-up clinical evaluation. Asymptomatic dengue infection was defined by the detection of DENV-specific IgM and no report of current or previous acute febrile syndrome between clinical evaluations [18].

\section{Statistical analysis}

STATA (version 12) software was used for the statistical analyses. To control the confounding effect of age in the data analysis, we estimated the DENV incidence (DENV infections per 1000 person years) and its association with risk factors by age bands through Poisson regression model for cohort studies. This methodology assumes a constant incidence in each age strata (bands), but distinct rates between them [24]. According to this model, the data related to each subject is divided into parts that refer to the follow up of each child in the cohort through a single age strata (age band) [24].

The frequency of DENV serotypes (detected by RT-PCR) by year in follow-up was described. The crude and adjusted risk ratios (RR) of the association between DENV infection in the child cohort and maternal (age, self-reported skin colour, schooling, family income, and maternal DENV immune status) and child (sex and exclusive breastfeeding) characteristics were estimated in two separate age bands $(0-12$ months and $>12$ months of age). All independent variables were maintained in the final multivariate model due to the low number of variables analysed.

The incidence rates of DENV infection in the first year of life was estimated by 2-month age bands and compared with the proportion of children with detectable levels of maternally transferred DENV-3 neutralizing antibodies (determined by the Kaplan Meyer curve) assessed in a subset of the child cohort (previously published, reference 20). Moreover, the unadjusted effect of each maternal DENV serotypespecific antibody on the incidence of dengue infection in the first 12 months of follow-up was estimated.

\section{RESULTS}

Overall, $85 \cdot 3 \%$ (354/415) of the infants enrolled at birth were examined at least once during the first year of life, resulting in a loss to follow-up rate of $14 \cdot 7 \%$. No losses to follow-up by death were observed in the study. The median follow-up period was 12 months (range 2-30 months). A total of 49 dengue infections were detected during follow-up: 21 by RT-PCR and 28 by IgM followed by IgG seroconversion. Of those, none progressed to severe disease. The incidence rate was $107 \cdot 6 / 1000$ person-years $(95 \%$ CI $76 \cdot 8-150 \cdot 6)$ and $93 \cdot 3 / 1000$ person-years $(95 \%$ CI $56 \cdot 1-154 \cdot 4)$ in the first and second years of life, respectively. Among the DENV infections detected, 29 were classified as symptomatic infections, yielding an asymptomatic/symptomatic ratio of $1: 1.45$ and an incidence rate of clinical disease of $60 \cdot 7 / 1000$ personyears $(95 \%$ CI $42 \cdot 2-87 \cdot 4)$ in the cohort. 
We reported 138 infants with acute undifferentiated febrile illness during follow-up. Among them, DENV infections were virologically confirmed in $15 \cdot 2 \%(n=$ 21) of the children. Other diagnosis included respiratory tract infections (34.4\%), roseola $(14 \cdot 3 \%)$ and ear infections $(11 \cdot 6 \%)$. DENV-1 $(n=2)$ and DENV-2 $(n=1)$ were the serotypes detected in the first year of study (2011). In 2012, all four DENV serotypes were identified: DENV-1 $(n=2)$, DENV-2 $(n=1)$, DENV-3 $(n=3)$, and DENV-4 $(n=6)$. In 2013/ 2014, DENV-1 $(n=2), \operatorname{DENV}-3(n=1)$ and DENV-4 $(n=3)$ were detected. DENV-4 was first detected in the study population in 2012 and was the predominant serotype from 2012 to 2014 (Fig. 1). None of the children were simultaneously or sequentially infected by two or more DENV serotypes.

Table 1 shows the association between maternal and child characteristics and dengue incidence by age bands ( $0-12$ and $>12-30$ months of age). In the $0-12$-month age band, the crude analysis of the risk factors showed a statistically significant association between both the maternal dengue immune status and child sex with DENV infection (Table 1). This statistical association remained in the multivariate analysis. Male children and those born to DENV-naïve mothers had a twofold higher risk of dengue infection.

In the 12-30-month age band, the self-reported maternal skin colour was associated with DENV infection in the crude analysis. This association remained after adjustment in the multivariate model. Children born to Caucasian/Asian descent skin colour mothers had a threefold higher risk of infection compared with children born to mixed/black skin colour mothers (Table 1). The association between maternal immune status and DENV incidence in the 1230 -month age band was not tested due to the lack of dengue infections in children born to DENV-naïve mothers in this age band.

A progressive increase in the incidence rate of DENV infection coincided with the decay of maternally transferred DENV-3 neutralizing antibodies during the first year of life. The highest incidence rate was observed in the age band of 10-12 months, when maternal dengue antibodies were no longer detected (Fig. 2). Table 2 shows the incidence rates of dengue infection in the child cohort and its association with each DENV serotype-specific antibody detected in the mothers. Children born to DENV-3 immune mothers had a $60 \%$ lower chance of infection compared with children born to DENV-3 naïve

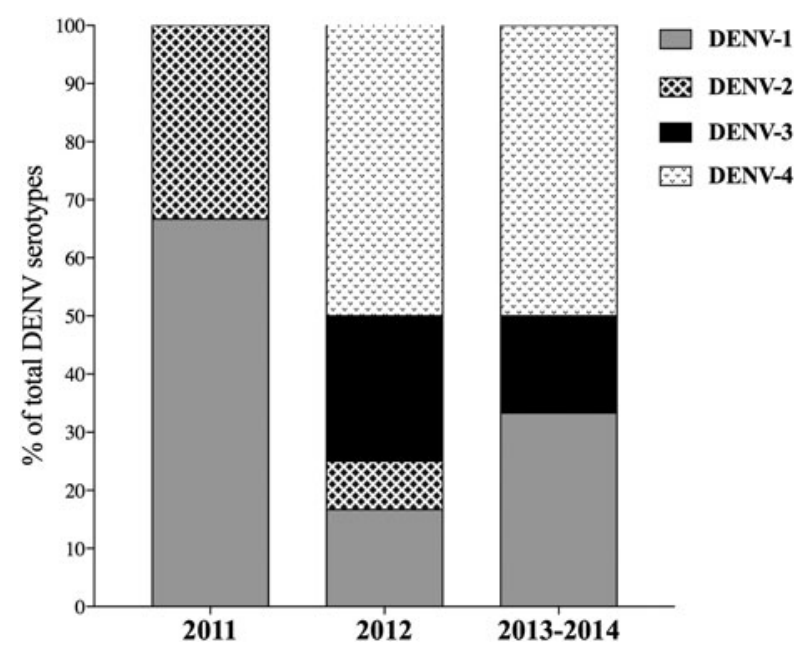

Fig. 1. Distribution of DENV serotypes detected during the study period. The percent of dengue infections by each DENV serotype in $2011(n=3), 2012(n=12)$ and 2013/14 $(n=6)$ are shown.

mothers (Table 2). There was no significant association between dengue incidence in children and maternal dengue immunity against DENV-1, DENV-2 and DENV-4. Moreover, there was no significant association between DENV maternal immune status (naïve, monotypic and multitypic) and dengue incidence in the children (Table 2).

\section{DISCUSSION}

Prospective birth cohort studies are invaluable tools to understand the transmission dynamic and pathogenesis of DENV infection at early ages [17]. These data are important for better defining surveillance activities, performing clinical management and establishing the appropriate age to initiate vaccination schedules [16, 17]. Here, we estimate the incidence of dengue infection and the risk factors associated with infection in Brazilian children living in a hyperendemic area.

We found a high dengue incidence in the first (107.6/1000 person-years) and second (93.3/1000 person-years) years of follow-up in our study population, suggesting an intense exposure to dengue infection among children in our setting. These rates are higher than the incidence observed in cohort studies conducted in paediatric populations from Asian countries, where severe dengue is a main cause of death and hospitalizations among children [7]. However, similar incidence rates have been reported in older children [25]. Few studies have estimated the incidence 
Table 1. Crude and adjusted incidence rate ratios (RR) of the association between maternal and child characteristics and dengue infection by age bands in the cohort

\begin{tabular}{|c|c|c|c|c|c|c|c|c|c|c|c|c|}
\hline \multirow[b]{3}{*}{ Characteristics } & \multicolumn{12}{|l|}{ Age bands } \\
\hline & \multicolumn{6}{|c|}{ 0-12 months } & \multicolumn{6}{|c|}{$>12-30$ months } \\
\hline & $\begin{array}{l}\text { DENV } \\
\text { infections }\end{array}$ & $\begin{array}{l}\text { Person-years } \\
\text { at risk }\end{array}$ & $\begin{array}{l}\text { Crude RR } \\
(95 \% \text { CI })\end{array}$ & $\begin{array}{l}P \\
\text { value }\end{array}$ & $\begin{array}{l}\text { Adjusted RR } \\
(95 \% \mathrm{CI})\end{array}$ & $\begin{array}{l}P \\
\text { value }\end{array}$ & $\begin{array}{l}\text { DENV } \\
\text { infections }\end{array}$ & $\begin{array}{l}\text { Person-years } \\
\text { at risk }\end{array}$ & $\begin{array}{l}\text { Crude RR } \\
(95 \% \text { CI })\end{array}$ & $\begin{array}{l}P \\
\text { value }\end{array}$ & $\begin{array}{l}\text { Adjusted RR } \\
(95 \% \mathrm{CI})\end{array}$ & $\begin{array}{l}P \\
\text { value }\end{array}$ \\
\hline \multicolumn{13}{|l|}{ Mothers } \\
\hline \multicolumn{13}{|l|}{ Age (in years) } \\
\hline$\geqslant 20$ & 10 & 0.097 & 1.00 & & 1.00 & & 5 & 0.051 & 1.00 & & 1.00 & \\
\hline$<20$ & 24 & 0.219 & $0.94(0.45-1.97)$ & 0.869 & $0.74(0.34-1.59)$ & 0.443 & 10 & 0.109 & $1.06(0.36-3.11)$ & 0.909 & $1.08(0.35-3.32)$ & 0.880 \\
\hline \multicolumn{13}{|l|}{$\begin{array}{l}\text { Self-reported skin } \\
\text { colour }\end{array}$} \\
\hline Mixed/black & 31 & 0.251 & 1.00 & & 1.00 & & 9 & 0.130 & 1.00 & & 1.00 & \\
\hline $\begin{array}{l}\text { Caucasian/Asian } \\
\text { descent }\end{array}$ & 3 & 0.064 & $0.37(0.11-1.23)$ & 0.108 & $0.35(0.10-1.17)$ & 0.090 & 6 & 0.031 & $2.80(1.00-7.86)$ & 0.051 & $3.34(1.08-10.33)$ & 0.036 \\
\hline \multicolumn{13}{|l|}{ Schooling (in years) } \\
\hline$\geqslant 9$ & 21 & 0.220 & 1.00 & & 1.00 & & 11 & 0.121 & 1.00 & & 1.00 & \\
\hline$<9$ & 13 & 0.095 & $1.43(0.71-2.86)$ & 0.310 & $1.44(0.69-2.98)$ & 0.321 & 4 & 0.039 & $1.11(0.35-3.48)$ & 0.857 & $1.32(0.38-4.56)$ & 0.654 \\
\hline \multicolumn{13}{|c|}{$\begin{array}{l}\text { Family income } \\
\text { (in minimum wages)* }\end{array}$} \\
\hline$>1$ & 17 & 0.193 & 1.00 & & 1.00 & & 10 & 0.104 & 1.00 & & 1.00 & \\
\hline$\leqslant 1$ & 13 & 0.093 & $1.58(0.77-3.26)$ & 0.212 & $0.47(0.59-2.64)$ & 0.557 & 5 & 0.044 & $1.17(0.40-3.43)$ & 0.769 & $1.42(0.45-4.43)$ & 0.654 \\
\hline \multicolumn{13}{|l|}{ DENV-specific IgG $\dagger$} \\
\hline Positive & 24 & 0.269 & 1.00 & & 1.00 & & 14 & 0.138 & - & & - & \\
\hline Negative & 7 & 0.034 & $2.30(1.00-5.31)$ & 0.054 & $2.42(1.01-5.80)$ & 0.047 & 0 & 0.018 & - & & - & \\
\hline \multicolumn{13}{|c|}{ (2) } \\
\hline \multicolumn{13}{|l|}{ Sex } \\
\hline Female & 10 & 0.163 & 1.00 & & 1.00 & & 8 & 0.084 & 1.00 & & 1.00 & \\
\hline Male & 24 & 0.152 & $2.57(1.23-5.38)$ & 0.012 & $2.33(1.09-4.98)$ & 0.028 & 7 & 0.077 & $0.94(0.34-2.60)$ & 0.911 & $0.66(0.22-1.92)$ & 0.449 \\
\hline \multicolumn{13}{|l|}{$\begin{array}{l}\text { Exclusive breastfeeding } \\
\text { (in months) }\end{array}$} \\
\hline$\geqslant 4$ & 11 & 0.152 & 1.00 & & 1.00 & & 8 & 0.079 & 1.00 & & 1.00 & \\
\hline$<4 /$ never breastfed & 23 & 0.162 & $1.97(0.96-4.04)$ & 0.064 & $1.87(0.90-3.88)$ & 0.093 & 7 & 0.081 & $0.86(0.31-2.38)$ & 0.773 & $0.99(0.35-2.81)$ & 0.990 \\
\hline
\end{tabular}

CI, confidence interval; $\mathrm{RR}$, rate ratios.

* 36 missing values.

$\dagger$ DENV-specific IgG was determined in the maternal samples by enzyme-linked immunosorbent assays (ELISA); 14 missing values. 


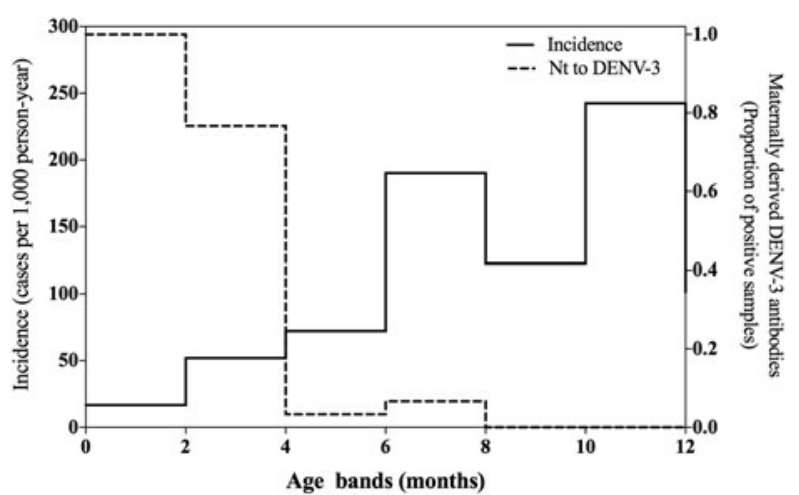

Fig. 2. Incidence of DENV infection and decay of DENV neutralizing antibodies by age bands during the first year of follow-up in the child cohort.

of dengue infection in children under 2-years old [26-29]. In Vietnam, a birth cohort study of 1244 infants found a dengue incidence of 17 cases $/ 1000$ person-years [27]. In the Philippines, a cohort study reported an incidence rate of 121.9 infections $/ 1000$ person-years between 6 months and 5 years of age in a 1-year-long follow-up [28]. Of note, most children included in our study belong to low-income families and were potentially more exposed to dengue infection than the population living in privileged areas, as previously reported in a dengue serosurvey conducted in our setting [12].

In contrast to reports from other studies conducted in Asian countries [7], none of the dengue infections detected in our cohort progressed to severe disease. The mechanisms underlying the immunopathogenesis of severe dengue in infants are complex and multifactorial, which includes serotype-specific differences in maternal exposure to DENV and the rate of decay of maternally transferred antibodies, virulence of the circulating DENV serotypes, and/or genetic background of the population $[6,21,30]$. These combined factors might explain the differences in the dengue severe rates among infants observed between Asia and Brazil [7]. In fact, we previously reported that enhancement of DENV infection by maternally derived dengue antibodies occurred at earlier ages (2-4 months) in our cohort [20] compared with Asian studies (6-9 months) [31]. Because severe dengue is typically seen in approximately $1 \%$ of dengue cases overall [32], we acknowledge that our cohort size was probably too small to detect severe cases.

All four DENV serotypes were observed in Recife during the study period. This finding agrees with the current epidemiological scenario of DENV circulation in the study setting [5, 20-22]. Of the four serotypes, DENV-3 was recognized as the predominant serotype circulating in the study area between 2002 and 2009. However, DENV-3 predominance was replaced by DENV-4 after its introduction in 2010 (Health Department of Pernambuco State, unpublished data). Of note, infections by DENV-3 and DENV-4 were not detected in the cohort during the first year of follow up, which was probably due to the high frequency of children with detectable levels of maternally transferred neutralizing antibodies to these DENV serotypes at birth [20].

Our results showed that male children had a twofold higher risk of dengue infection than females during the first year, but not in the second year of life. Studies investigating sex differences for DENV infection in the paediatric population are scarce. In adults, higher infection rates in males than females have been described, which probably reflect sex-related differences in health-seeking behaviour [33, 34]. This latter hypothesis may not be plausible for infants in our setting. The mechanisms underlying sex differences in the early ages are probably multifactorial and might include endocrine and genetic effects on the immune system and physiology, which probably explains the higher vulnerability of the male sex to infections [35]. Additionally, sex differences in the maturation of the immunological system during infancy [35] might contribute to the higher risk for dengue infection among males observed in the first year and the disappearance of this risk in the second year. Although our results agree with previous studies that showed higher vulnerability of male children to several paediatric exanthematic viral diseases, including rubella and measles [35, 36], this finding of different risk of dengue infection by sex must be confirmed in others paediatric cohort studies.

In the second year of life, Caucasian/Asian descent self-reported maternal skin colour was a predictor for dengue infection in the cohort. Differences in the severity and incidence of dengue cases between races have been described by several studies, especially in Caribbean countries [11]. In Cuba, a significantly higher proportion of whites than blacks developed severe dengue during the 1981 dengue epidemic. Among children diagnosed with severe disease during this epidemic, $86 \%$ of the cases were recorded among self-declared white individuals and $7 \%$ in individuals with a black skin colour [37]. These distinct clinical outcomes among individuals from different races probably reflect the complex interactions between 
Table 2. Crude incidence rate (per 1000 person-year) and rate ratios of the association between maternal dengue immunity and dengue infection in the first year of follow-up

\begin{tabular}{|c|c|c|c|c|c|}
\hline $\begin{array}{l}\text { Maternal dengue } \\
\text { immunity* }\end{array}$ & $\begin{array}{l}\text { DENV } \\
\text { infections }\end{array}$ & $\begin{array}{l}\text { Person-years } \\
\text { at risk }\end{array}$ & $\begin{array}{l}\text { Crude Incidence rate per } \\
1000 \text { person-years }(95 \% \mathrm{CI})\end{array}$ & $\begin{array}{l}\text { Rate ratio } \\
(95 \% \mathrm{CI})\end{array}$ & $P$ value \\
\hline \multicolumn{6}{|l|}{ DENV-1 } \\
\hline Negative & 33 & $0 \cdot 289$ & $114 \cdot 22(81 \cdot 20-160 \cdot 66)$ & - & \\
\hline Positive & 0 & $0 \cdot 173$ & - & - & - \\
\hline \multicolumn{6}{|l|}{ DENV-2 } \\
\hline Negative & 31 & $0 \cdot 291$ & $106 \cdot 53(74.92-151 \cdot 48)$ & $1 \cdot 00$ & \\
\hline Positive & 1 & $0 \cdot 014$ & $69 \cdot 41(9 \cdot 77-492 \cdot 76)$ & $0.65(0.09-4.77)$ & 0.673 \\
\hline \multicolumn{6}{|l|}{ DENV-3 } \\
\hline Negative & 8 & 0.037 & $212 \cdot 40(106 \cdot 22-424 \cdot 72)$ & $1 \cdot 00$ & \\
\hline Positive & 25 & $0 \cdot 269$ & $92 \cdot 74(62 \cdot 66-137 \cdot 25)$ & $0.44(0.20-0.97)$ & $0 \cdot 041$ \\
\hline \multicolumn{6}{|l|}{ DENV-4 } \\
\hline Negative & 22 & $0 \cdot 202$ & $108 \cdot 67(71 \cdot 56-165 \cdot 05)$ & $1 \cdot 00$ & \\
\hline Positive & 10 & $0 \cdot 104$ & $95 \cdot 74(51 \cdot 51-177.93)$ & $0 \cdot 88(0 \cdot 41-1 \cdot 86)$ & $0 \cdot 740$ \\
\hline \multicolumn{6}{|l|}{ DENV immunity } \\
\hline Multitypic & 10 & $0 \cdot 115$ & $86.89(46 \cdot 75-161 \cdot 49)$ & $1 \cdot 00$ & \\
\hline Monotypic & 14 & $0 \cdot 154$ & $90 \cdot 55(53 \cdot 63-152 \cdot 89)$ & $1 \cdot 04(0 \cdot 46-2 \cdot 35)$ & 0.921 \\
\hline Naïve & 7 & 0.034 & $203 \cdot 77(97 \cdot 15-427 \cdot 44)$ & $2 \cdot 35(0 \cdot 90-6 \cdot 16)$ & $0 \cdot 084$ \\
\hline
\end{tabular}

CI, confidence interval.

* Maternal serotype-specific (DENV1-4) dengue immunity was determined by plaque reduction neutralization test.

viral and genetic factors in determining the dengue pathogenesis and vulnerability to infection.

Overall, there is a lack in clearly defined sociodemographic risk factors associated with dengue infection in infants. Alternatively, the protective role of the maternally transferred antibodies in shaping dengue disease at the first months of life has been confirmed by different cohort studies performed in Asian countries [15, 26, 27, 38]. In our study, the incidence rate of dengue infection among children increased as the maternally transferred DENV-3 antibodies waned to undetectable levels. One caveat of this analytical approach is that the rise of dengue infection incidence rates in the cohort as a whole was compared with the decay of maternal transferred dengue antibodies in a subgroup of infants. However, the association between maternal immunity and dengue infection at early ages is confirmed at an individual level, since we observed that infants born to mothers with pre-existing immunity against DENV-3 were at a lower risk of infection during the first year of life.

The main limitation of our study is the relatively small sample size of dengue infections used to perform inferences about risk factors, which may have resulted in a lower power of the study to detect small differences in the incidence rates among some of the risk factors analysed in our study. We acknowledge that the sample size for this study was primarily calculated to estimate the overall incidence of dengue infection in the cohort [18]. However, even considering the lack of power to detect smaller differences in the incidence rates, our study was able to identify statistically significant risk factors associated with dengue infection in children. These associations could probably be confirmed by a large sample size for the dengue-infected group, consequently leading to exponentially more power. However, it is noteworthy to emphasize the operational difficulties in follow-up of a large sample of children of early age in which repeated blood samples are collected.

In summary, we demonstrated a high incidence of DENV infection among Brazilian children living in a hyperendemic area, indicating the high exposure of the population to infection at early ages in this setting. Currently, the only licensed dengue vaccine has shown an increased efficacy when administered in individuals that have been exposed to at least one DENV serotype [39]. In Brazil, this vaccine is licensed for use in children with 9 years and older. Taken into account the relatively high incidence of dengue infections found in our cohort study, the implementation of dengue vaccines would probably be better directed at early age groups ( $>1$ year), since their incidence and prevalence of disease are comparable with those observed in adults. However, considering that dengue attack rates 
vary significantly across settings [5, 12, 21, 22], it is important to define the age-specific dengue seroprevalence in each region in order to make the best possible vaccine recommendation. Moreover, surveillance strategies to monitor the incidence of dengue and oscillations in the circulating serotypes might be directed to these age groups. Finally, stimulation of parental behaviour that reduces the exposure of children to mosquito bites, including the use of bed nets and repellents, might contribute to lower DENV infection rates in children at early ages.

\section{ACKNOWLEDGEMENT}

We thank the mothers and their infants, for participating in the study; and the nurses and clinical staff from IMIP who assisted with the study. This work was supported by the National Council for Scientific and Technological Development of the Brazilian government (C.B., grant 482915/2010-2 MCT/CNPq-14/ 2010); and the Strategic Program to Support Health Research/PAPES VI (C.B., grant 407697/2012-8).

\section{DECLARATION OF INTEREST}

None declared.

\section{REFERENCES}

1. Guzman MG, Harris E. Dengue. Lancet 2015; 385: 453-465.

2. World Health Organization. Dengue and severe dengue. Fact sheet no. 117 (http://www.who.int/mediacentre/ factsheets/fs117/en/). Accessed 7 July 2016.

3. Bhatt S, et al. The global distribution and burden of dengue. Nature 2013; 496: 504-507.

4. San Martín JL, et al. The epidemiology of dengue in the Americas over the last three decades: a worrisome reality. The American Journal of Tropical Medicine and Hygiene 2010; 82: 128-135.

5. Teixeira MG, et al. Epidemiological trends of dengue disease in Brazil (2000-2010): a systematic literature search and analysis. PLoS Neglected Tropical Diseases 2013; 7: e2520.

6. Jain A, Chaturvedi UC. Dengue in infants: an overview. FEMS Immunology \& Medical Microbiology 2010; 59: 119-130.

7. Halstead SB, et al. Dengue hemorrhagic fever in infants: research opportunities ignored. Emerging Infectious Diseases 2002; 8: 1474-1479.

8. Verhagen LM, de Groot R. Dengue in children. Journal of Infection 2014; 69: S77-S86.

9. Thai KT, et al. Seroprevalence of dengue antibodies, annual incidence and risk factors among children in southern Vietnam. Tropical Medicine \& International Health 2005; 10: 379-386.

10. Teixeira MG, et al. Risk factors for the incidence of dengue virus infection in preschool children. Tropical Medicine \& International Health 2012; 17: 1391-1395.

11. de la C Sierra B, Kourí G, Guzmán MG. Race: a risk factor for dengue hemorrhagic fever. Archives of Virology 2007; 152: 533-542.

12. Braga $\mathbf{C}$, et al. Seroprevalence and risk factors for dengue infection in socio-economically distinct areas of Recife, Brazil. Acta Tropica 2010; 113: 234-240.

13. Endy TP, et al. Relationship of preexisting dengue virus (DV) neutralizing antibody levels to viremia and severity of disease in a prospective cohort study of DV infection in Thailand. The Journal of Infectious Diseases 2004; 189: 990-1000.

14. Argüello DF, et al. Incidence of dengue virus infection in school-aged children in Puerto Rico: a prospective seroepidemiologic study. The American Journal of Tropical Medicine and Hygiene 2015; 92: 486-491.

15. Libraty DH, et al. A prospective nested case-control study of dengue in infants: rethinking and refining the antibody-dependent enhancement dengue hemorrhagic fever model. PLoS Medicine 2009; 6: e1000171.

16. Endy TP, Yoon IK, Mammen MP. Prospective cohort studies of dengue viral transmission and severity of disease. Current Topics in Microbiology and Immunology 2010; 338: 1-13.

17. Endy TP. Human immune responses to dengue virus infection: lessons learned from prospective cohort studies. Frontiers in Immunology 2014; 5: 183.

18. Braga C, et al. Prospective birth cohort in a hyperendemic dengue area in Northeast Brazil: methods and preliminary results. Cadernos de Saude Publica 2016; 32: $\mathrm{e} 00095815$.

19. Leite RC, et al. Dengue infection in pregnancy and transplacental transfer of anti-dengue antibodies in northeast, Brazil. Journal of Clinical Virology 2014; 60: $16-21$.

20. Castanha PM, et al. Placental transfer of dengue virus (DENV)-specific antibodies and kinetics of DENV infection-enhancing activity in Brazilian infants. The Journal of Infectious Diseases 2016; 214: 265-272.

21. Cordeiro MT, et al. Characterization of a dengue patient cohort in Recife, Brazil. The American Journal of Tropical Medicine and Hygiene 2007; 77: 1128-1134.

22. Castanha PM, et al. Force of infection of dengue serotypes in a population-based study in the northeast of Brazil. Epidemiology and Infection 2013; 141: 1080 1088.

23. Roehrig JT, Hombach J, Barrett AD. Guidelines for plaque-reduction neutralization testing of human antibodies to dengue viruses. Viral Immunology 2008; 21: 123-132.

24. Clayton D, Hills M. Poisson and logistic regression. In: Clayton D, Hills M, eds. Statistical Models in Epidemiology. New York: Oxford University Press, 1993. pp. 3421-3452.

25. Gordon A, et al. The nicaraguan pediatric dengue cohort study: incidence of inapparent and symptomatic 
dengue virus infections, 2004-2010. PLoS Neglected Tropical Diseases 2013; 7: e2462.

26. Pengsaa K, et al. Dengue virus infections in the first 2 years of life and the kinetics of transplacentally transferred dengue neutralizing antibodies in Thai children. The Journal of Infectious Diseases 2006; 194: 15701576.

27. Chau TN, et al. Dengue virus infections and maternal antibody decay in a prospective birth cohort study of Vietnamese infants. The Journal of Infectious Diseases 2009; 200: 1893-1900.

28. Alera MT, et al. Incidence of dengue virus infection in adults and children in a prospective longitudinal cohort in the Philippines. PLoS Neglected Tropical Diseases 2016; 10: e0004337.

29. Clapham H, et al. Epidemiology of infant dengue cases illuminates serotype-specificity in the interaction between immunity and disease, and changes in transmission dynamics. PLoS Neglected Tropical Diseases 2015; 9: $\mathrm{e} 0004262$.

30. Ribeiro CF, et al. Perinatal transmission of dengue: a report of 7 cases. The Journal of Pediatrics 2013; 163: 1514-1516.

31. Chau TN, et al. Dengue in Vietnamese infants -results of infection-enhancement assays correlate with age-related disease epidemiology, and cellular immune responses correlate with disease severity. The Journal of Infectious Diseases 2008; 198: 516-524.
32. Corbett KS, et al. Preexisting neutralizing antibody responses distinguish clinically inapparent and apparent dengue virus infections in a Sri Lankan pediatric cohort. The Journal of Infectious Diseases 2015; 211: 590-599.

33. Anker M, Arima Y. Male-female differences in the number of reported incident dengue fever cases in six Asian countries. Western Pacific Surveillance Response Journal 2011; 2: 17-23.

34. Raza FA, et al. Demographic and clinico-epidemiological features of dengue fever in Faisalabad, Pakistan. PLoS ONE 2014; 9: e89868.

35. Muenchhoff M, Goulder PJ. Sex differences in pediatric infectious diseases. The Journal of Infectious Diseases 2014; 209: 120-126.

36. Del Principe D, et al. Gender disparity in pediatric diseases. Current Molecular Medicine 2013; 13: 499-513.

37. Bravo JR, Guzmán MG, Kouri GP. Why dengue haemorrhagic fever in Cuba? 1. Individual risk factors for dengue haemorrhagic fever/dengue shock syndrome (DHF/DSS). Transactions of the Royal Society of Tropical Medicine and Hygiene 1987; 81: 816-820.

38. Kliks SC, et al. Evidence that maternal dengue antibodies are important in the development of dengue hemorrhagic fever in infants. The American Journal of Tropical Medicine and Hygiene 1988; 38: 411-419.

39. Villar L, et al. Efficacy of a tetravalent dengue vaccine in children in Latin America. The New England Journal of Medicine 2015; 372: 113-123. 